Vol. 6, Num. 2, 2021

\title{
METHODS TO INCREASE THE CONTRAST OF THE IMAGE WITH PRESERVING THE VISUAL QUALITY
}

\author{
Mykola Maksymiv ${ }^{1}$, Taras Rak ${ }^{2}$ \\ ${ }^{1,2}$ Lviv Polytechnic National University, 12, Bandera Str, Lviv, 79013, Ukraine \\ ${ }^{2}$ IT STEP University, 83a, Zamarstynivska Str, Lviv, 79019, Ukraine. \\ Author's e-mail: mykola.maksymiv.mki.2020@lpnu.ua
}

Submitted on 13.05.2021

(C) Maksymiv M., Rak T., 2021

\begin{abstract}
Contrast enhancement is a technique for increasing the contrast of an image to obtain better image quality. As many existing contrast enhancement algorithms typically add too much contrast to an image, maintaining visual quality should be considered as a part of enhancing image contrast.

This paper focuses on a contrast enhancement method that is based on histogram transformations to improve contrast and uses image quality assessment to automatically select the optimal target histogram. Improvements in contrast and preservation of visual quality are taken into account in the target histogram, so this method avoids the problem of excessive increase in contrast. In the proposed method, the optimal target histogram is the weighted sum of the original histogram, homogeneous histogram and Gaussian histogram. Structural and statistical metrics of "naturalness of the image" are used to determine the weights of the corresponding histograms. Contrast images are obtained by matching the optimal target histogram. Experiments show that the proposed method gives better results compared to other existing algorithms for increasing contrast based on the transformation of histograms.
\end{abstract}

Index Terms: Digital images, Image enhancement, Image processing, Image quality, Histograms.

\section{INTRODUCTION}

Due to the popularity of professional digital cameras and the proliferation of smartphones with high-quality cameras, it has become extremely easy and convenient to take photos. However, some images obtained by such devices are not good enough in terms of visual quality. This can be explained by various reasons and factors: poor lighting, poor smartphone camera, poorly chosen camera settings, and so on.

The contrast enhancement of image refers to the amount of color or gray differentiation that exists between various features in digital images. It is the range of the brightness present in the image. The images having a higher contrast level usually display a larger degree of color or gray scale difference as compared to lower contrast level. The contrast enhancement is a process that allows image features to show up more visibly by making best use of the color presented on the display devices. During last decade a number of contrast enhancement algorithms have been developed for the contrast enhancement of images for various applications.

Accordingly, the task of developing methods to improve image quality is urgent. Image quality enhancement is used in various areas where image processing takes place and where critical decisions can be made based on images, such as camera photography [1], medical imaging [2], remote sensing imaging [3], etc. One approach to improving image quality is to work with contrast.

To increase the contrast of the image, many different methods have been developed [4]. One of the most common and popular are methods based on the transformation of histograms.

A histogram is a chart constructed in a columnar form, in which the value of the indicator is depicted graphically in the form of a column. The histogram clearly characterizes how the value of the indicator changes over time. In our case, the histogram shows the brightness level of individual image elements. Optimal for human visual perception is the image, the elements of which have a uniform distribution of brightness levels. Improving images by aligning the histogram is a process in which they try to achieve a uniform distribution of the brightness of the processed image. The histogram alignment procedure involves the following sequence of actions:

- calculates the histogram of the brightness distribution of the image elements $H(L)$;

- normalized cumulative histogram $\mathrm{CH}(\mathrm{L})$ is constructed;

- a new image $L^{\prime}=R * C H(L)$ is formed.

This conversion is effective for improving the visual quality of low-contrast parts.

The described histogram conversion method can be global, i.e., use information about the whole image, and local, when local image areas are used for conversion. Global methods of histogram transformation are, in fact, tabular methods, their main advantage is speed. Local methods are used for more detailed image processing. They provide highquality contrast of small details of the image. However, they are more voluminous with the so-called computational complexity. Therefore, when using the methods of the class of histogram transformations, it is necessary to look for a compromise between the quality and speed of image processing [5]. Quite often, the image is distorted only in certain places, which are caused by diffraction of light, shortcomings of optical systems or defocusing. This leads to the need to perform local transformations of the certain blocks of the image. In this approach, the informative areas in the 
image are highlighted and processed accordingly. The main steps of the method are [6]:

1. For each image element $L(i, j)$ calculate the value of the local contrast $C(i, j)$ in the current area $\mathrm{W}$ with the center in the element with coordinates $(i, j)$.

2. Calculate local statistics for the current area $W$.

3. Transform (amplify) the local contrast $C(i, j)$ using nonlinear functions and taking into account the local statistics of the current section $W$.

4. Restore the brightness of the image $L^{\prime}(i, j)$ with enhanced local contrast.

This method can be improved by calculating the local contrast around each pixel using adjacent pixels, the size of which depends on the statistical properties around the pixel. The resulting contrast image is then converted to a new contrast image using the contrast enhancement function. Finally, improved image contrast is obtained by applying the inverse contrast conversion to the previous step. This technique provides the benefits of enhancing or maintaining image contrast while suppressing noise.

Although local methods can usually get good results with careful parameter adjustment, their effectiveness largely depends on the appropriate parameter settings. Unsuccessful selection of parameters will lead to the fact that the artifacts will impair the visual quality of the images. One of the most popular global methods of improving contrast is the alignment of histograms, which modifies different types of histograms for homogeneity and creates a higher visual contrast. For some color images, the traditional alignment of histograms will lead to unnatural artifacts (excessive contrast enhancement) and change the original hue (Fig. 1.).
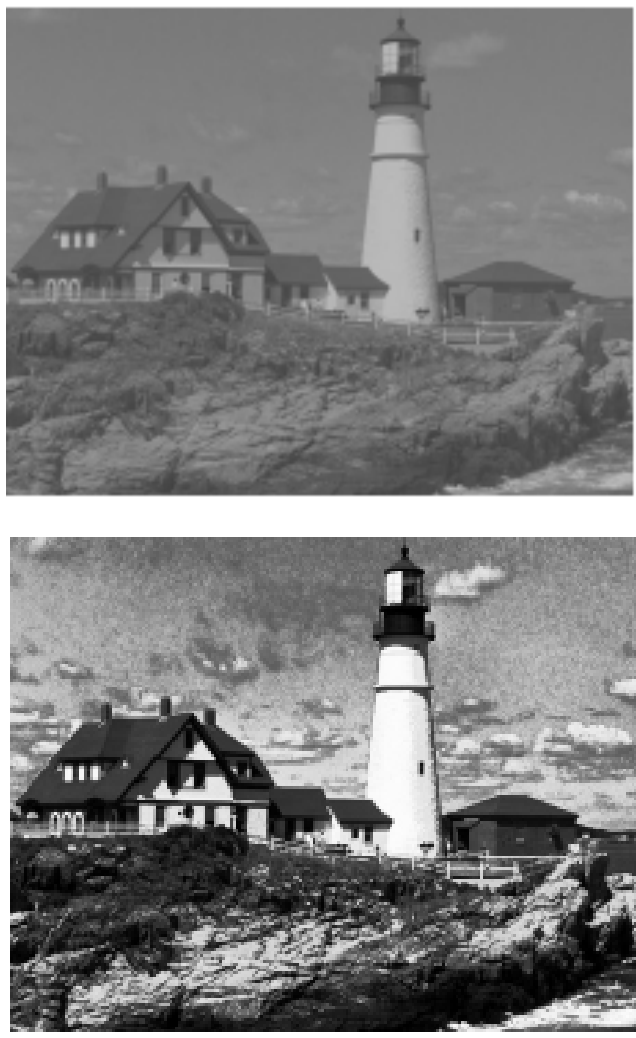

Fig. 1. Traditional photo contrast alignment (top) and by histogram for color image (bottom).

\section{RELATED WORKS}

To avoid such shortcomings, various related methods based on histograms have been proposed. In particular, J. A. Stark [7] proposed an adaptive method of image contrast enhancement (CLAHE) based on the generalization of histogram alignment (HE).

Methods based on histogram:

Histogram Equalization (hereinafter HE) is one of the typical methods of improvement in spatial area methods by the method based on global histograms. This technique is commonly used to improve an image because of its simplicity and relatively better performance on almost all types of images. The HE operation is performed by re-displaying the gray image levels based on the probability distribution of the input gray levels (Fig. 2.).

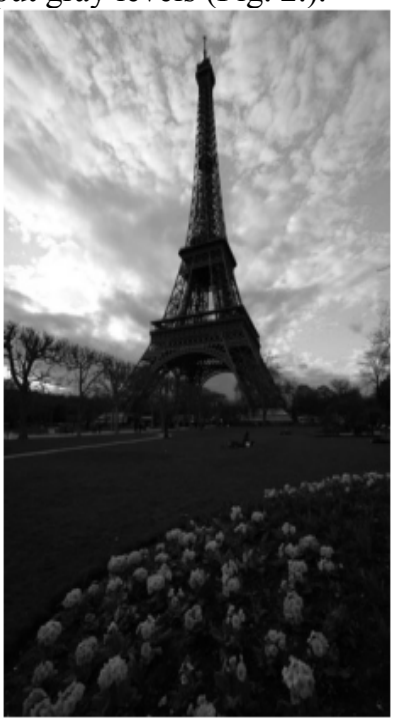

(a) Original image

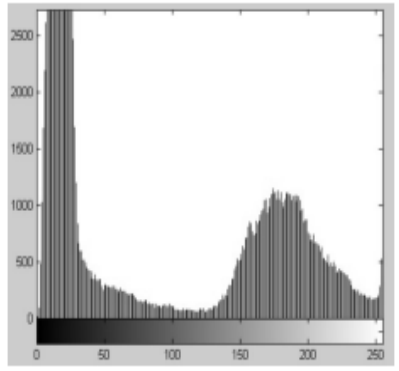

(c) Histogram of (a)

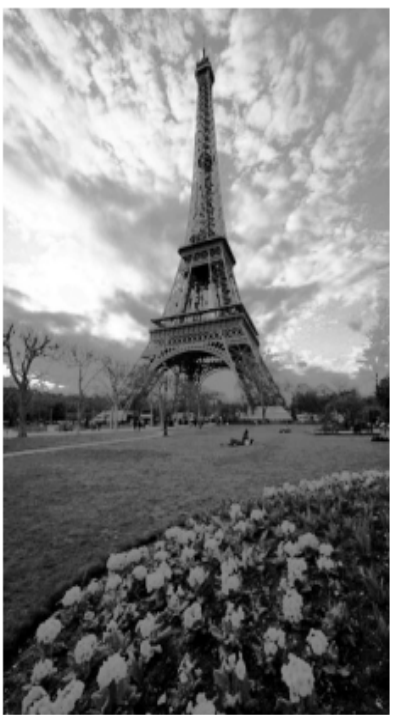

(b) Histogram Equalization

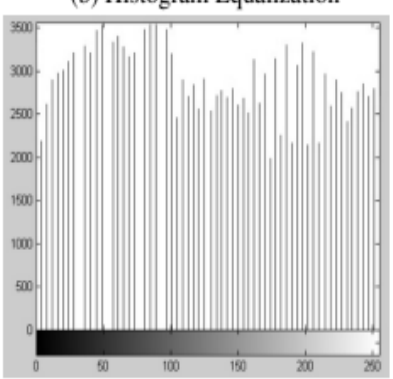

(d) Histogram of (b)

\section{Fig. 2. An example of histogram equalization.}

Consider a discrete image in shades of gray $\{x\}$ and let $n_{i}$ be the number of gray level inputs $i$. The probability of a pixel of level $i$ in the image is equal to [8]:

$$
p_{x}=\frac{n_{i}}{N}, 0<\mathrm{i}<\mathrm{L}
$$

Where, $L$ is the total number of gray levels in the image (usually 256), $N$ is the total number of pixels in the image, $p\{x\}(i)$ is the histogram of the image for the pixel value $i$

The HE method has quite serious disadvantages, especially when working with digital images, and conventional HE does not work with images that have more homogeneous areas of the image. To overcome the limitations 
of HE, many advanced methods of increasing contrast based on histograms have been investigated, but most of them are a modified version of the traditional HE technique.

The HE [9] method is based on global histograms. There are a number of advanced algorithms based on local histograms.

During the last decade a number of techniques have been proposed by various researchers to deal with these problems. In [10], the histogram is divided into two parts based on the input mean, and each part is equalized separately. This preserves the mean value of image to a certain extent. In [11], each peak of the histogram is equalized separately. An adaptation of HE, termed as Contrast Limited Adaptive Histogram Equalization (CLAHE), divides the input image into a number of equal sized blocks and then performs contrast limited histogram equalization on each block. The contrast limiting is done by clipping the histogram before histogram equalization. This tends to tone down the over enhancement effect of histogram equalization and gives a more localized enhancement. However, it is much more computationally intensive than histogram equalization. If the blocks are nonoverlapping, an interpolation scheme is needed to prevent blocky artifacts in the output picture. Therefore, overlapping blocks can solve this problem (every pixel is replaced by the histogram equalization output using a neighborhood) but it is more computationally intensive than using non-overlapping blocks. So, the CLAHE also requires a field store.

Adaptive Contrast Alignment with Limited Contrast (CLAHE) [12] is a traditional local histogram-based improvement algorithm. It considers the alignment of histograms in each local region. In other words, CLAHE defines a local conversion function for each local area based on the pixel distribution. This method is able to obtain more detailed information than global methods in order to control all the brightness and preserve the naturalness of the improved results.

Benefits:

- CLAHE was developed to prevent excessive noise amplification, which can lead to adaptive histogram alignment (AHE) [13][14];

- able to increase the contrast more than other methods.

Disadvantages:

- works on small areas of data (so-called tiles into which the image is divided), and not on the whole image;

- $\quad$ high computational complexity;

- high hardware complexity;

- a large number of operations;

- time complexity, because the algorithm that underlies the CLAHE method performs sequential recursive processing of local image blocks, when HE process image globally [15].

\section{FORMULATION OF THE PROBLEM}

The aim of the article is to describe the method of improving the contrast of the image by selecting the best target histogram based on a statistical measure of "naturalness" (visual quality) of the image.

\section{IV.METHOD FOR IMPROVING THE CONTRAST OF THE IMAGE}

The method of increasing the contrast while preserving the visual quality is proposed, which is shown in Fig. 3. The low-contrast image $I$ (RGB color space) is firstly converted to the full range of values $[0,255]$ with linear stretching. Then the stretched image is converted into HSV color space. The Vchannel was improved using histogram matching methods. Different target histograms can create different improved Vm. The optimal target histogram can be obtained from a structural measure (between $\mathrm{Vm}$ and $\mathrm{Vl}, \mathrm{Vl}$ is an improved result due to adaptive alignment of the histogram at the contrast boundary) and measurement of "naturalness" (for $V m$ ). After calculating the expanded $V e$ with the optimal target histogram, the final result of the $F$ improvement is produced by converting the HSV-RGB color space.

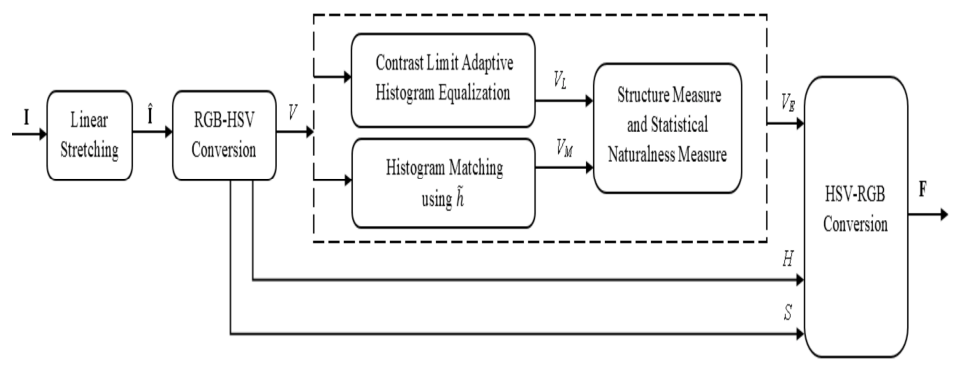

Fig. 3. General diagram of the proposed method of improving the contrast of the image

Since linear stretching, RGB-HSV conversion and HSVRGB conversion are the main methods of image processing [9], we will not provide a detailed description of these operations. In this section, we present a method for obtaining an extended $V e$ with an optimal histogram of the target and an intensity channel $V$. The key contributions are the determination of the target histogram and the calculation of the optimal weights of the components of the target histogram. Given the gray image $V$ with a total of $N$ pixels, the normalized histogram is defined as [16] $h_{v}(k)=\frac{n_{k}}{N}$ where $\mathrm{k}=[0,255]$ is the value of pixels, $\mathrm{nk}$ is the total number of pixels with the same value of $k$.

Contrast-enhancing methods based on histogram conversion use different types of histograms to obtain appropriate display functions to change the pixel values and increase the contrast of the considered image.

The traditional histogram alignment algorithm uses the original histogram of the considered image to obtain a display function. It tries to create a single histogram for the improved image, considering the aggregate histogram as the appropriate display function. Such a histogram alignment algorithm often causes problems of excessive improvement. To avoid this problem, T. Arici and others took into account the weight sum of the original histogram and homogeneous histogram [16].

A weighted histogram is used to create a display function. The problem of over-improvement can be reduced to some extent. Consider a more complex modification of the histogram, which is the weight sum of the original histogram, homogeneous histogram and histogram in the form of Gauss. In [17], the authors analyzed about 3,000 gray images and 
built a statistical model of "naturalness". They found that the histograms of these images could be well adjusted using the Gaussian function. A similar conclusion can also be found in [12], which takes a Gaussian curve to estimate well-exposed pixels.

Therefore, we take a Gaussian histogram to make the improvement more natural.

Based on the above analysis, the modified histogram $h$ should be closer to the normalized homogeneous histogram $h_{u}$ and the normalized histogram of the Gaussian form $h_{g}$, the residual $h-h_{v}$ should also be small. We define the target histogram $h^{\sim}$, which can be formulated as a minimization problem [9]:

where:

$$
h^{\sim}=\operatorname{argmin}(Z) \text {. }
$$

$Z=(1-\alpha-\beta)|| h-h_{v}\left\|2^{2}+\alpha\right\| h-h_{u}\left\|2^{2}+\beta\right\| h-h_{g} \| 2^{2}$

where $h_{u}$ is a normalized homogeneous histogram, $h_{g}$ is a normalized histogram with the shape of a Gaussian curve, $\alpha$ and $\beta$ are the respective weights, $\alpha+\beta=[0,1]$.

The Gaussian curve is used in the implementation. In general, $\alpha$ controls the enhancement of contrast, and $\beta$ controls the preservation of "naturalness".

First of all, we use the adaptive alignment of the histogram of the marginal contrast (CLAHE), which can create a satisfactory local structure of the image. We will get the result of improving CLAHE $V l$. At the next stage, we apply the statistical indicator of "naturalness" to achieve the preservation of the natural quality of the image. The optimal target histogram can be obtained from a structural measure (between $V m$ and $V l$ ). By measuring the "naturalness" (for $V m$ ) we can choose the optimal target histogram. After determining the target histogram, you can find the corresponding optimal result of $V e$ improvement.

\section{EXPERIMENTS}

The experiment used a traditional histogram alignment algorithm (HE), adaptive contrast margin alignment (CLAHE), histogram modification frame (HMF), and a proposed method to increase contrast while maintaining natural contrast in five test images from the image data set TID2013 [14] and CSIQ. Test images and corresponding reference images are available in these datasets. Test images with low contrast were obtained from the corresponding reference images with high contrast. Reference images are also provided to evaluate the operation of the algorithm.

For the traditional histogram alignment algorithm, the histeq function is used in the MATLAB image processing tool. The adapthisteq function in the MATLAB image processing tool is used for the adaptive alignment algorithm of the contrast boundary histogram. For the method of modification of the histogram, this algorithm is reproduced and the parameter $\lambda=1$ is set for all experiments [15]. The proposed method is implemented using MATLAB.

For all algorithms, the test image is first converted from the RGB color space to the HSV color space. The V-channel is then processed using appropriate enhancement algorithms.
Finally, the enhanced HSV image is converted to RGB color space.

\section{A. EFFICIENCY CRITERIA}

The quality of contrasting images can be assessed in many ways. Performance metrics used in our experiments: peak signal-to-noise ratio (PSNR) and structural similarity index (SSIM) [18], which are the most popular metrics for assessing image quality and comparing the performance of different image enhancement algorithms. The experiment used metric PSNR to measure the accuracy of the pixel value between the enhanced images and the corresponding highquality reference images and metric SSIM to measure the fidelity of the structure between the enhanced images and the reference images.

\section{B. ANALYSIS OF RESULTS}

To assess the effectiveness of the proposed method of improvement, both qualitative comparisons and quantitative assessments are considered.

\section{1) Qualitative comparisons}

Quantitative image analysis involves utilizing digital images to provide data and information. This is done with computer technology to recognize patterns, create maps, and process signals within images that cannot be done with the human eye due to the large amount of information generated and collected.

Some test images and the corresponding enhanced images were used to qualitatively compare the different enhancement algorithms. The original images are also provided to assess whether the corresponding results are excessive. In Fig. 4. HE makes histograms of improved results as homogeneous as possible. Some regions become lighter or darker, making the results unnatural. These problems can be found in the area of the door and the white ball (Fig. 4.a), the sky (Fig. 5.a), the fruit areas (Fig. 6.a). Comparing the average value or these images, our method (33.58) worked better by 79\% in PSNR than HE (18.68).

The average results of CLAHE (25.86) and HMF (26.84) to some extent avoid the problem of over-amplification (unnaturalness), but a small problem of unnaturalness can be observed. The reason is that these two methods do not take into account the preservation of naturalness. Comparing our results with others (better than CLAHE for 30\% and HMF for $25 \%$ ), we can conclude that the proposed method better preserves naturalness.

This conclusion can also be confirmed by comparing the results of the improvement with high-quality reference images. More comparisons of the results are shown in Fig. 5., Fig. 6.

\section{2) Quantitative comparisons}

To quantify the different enhancement algorithms, the two most popular image quality assessment metrics (PSNR and SSIM) are discussed below.

Peak signal-to-noise ratio is denoted by the abbreviation PSNR and is an engineering term meaning the relationship between the maximum possible signal value and the noise power that distorts the signal value. 


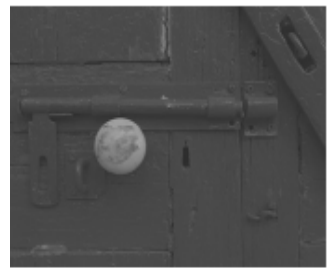

(a) Test Image

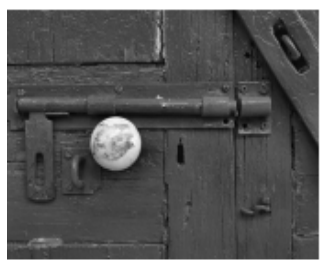

(c) CLAHE

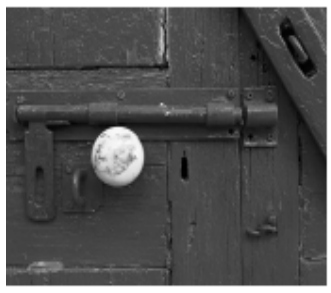

(e) Proposed

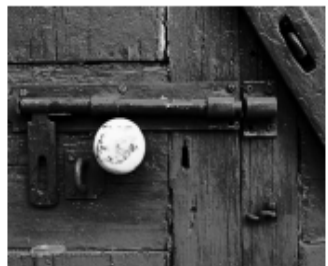

(b) HE

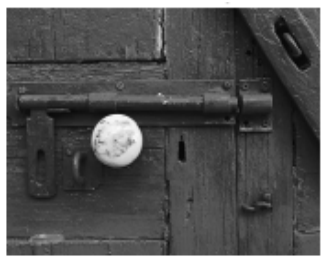

(d) HMF

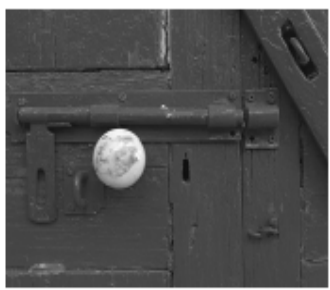

(f) Reference
Fig. 4. The results of algorithms: (b) Histogram equalization,

(c) Adaptive contrast margin alignment, (d) Histogram modification frame, (e) Proposed method - with improvement for image "Doors" based on (a) test image and comparison with $(f)$ reference image

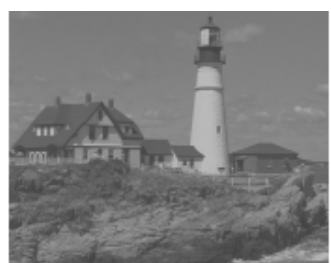

(a) Test Image

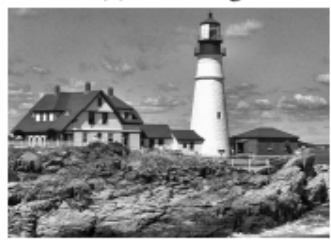

(c) CLAHE

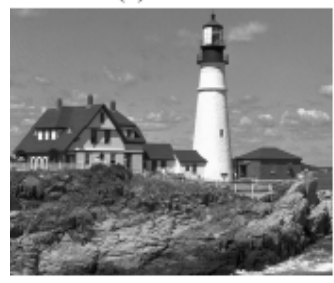

(e) Proposed

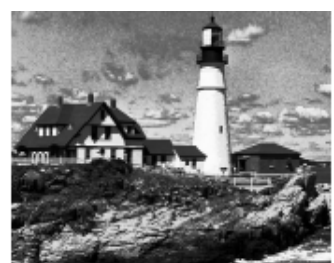

(b) HE

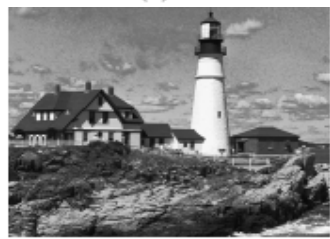

(d) HMF

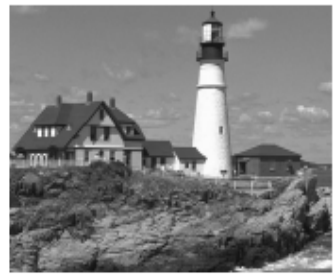

(f) Reference
Fig. 5. The results of algorithms: (b) Histogram equalization,

(c) Adaptive contrast margin alignment, (d) Histogram modification frame, (e) Proposed method - with improvement for image "Lighthouse" based on (a) test image and comparison with (f) reference image

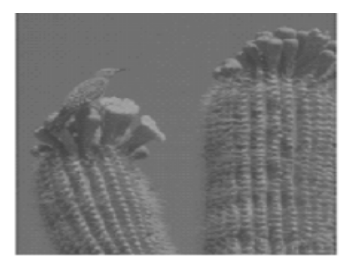

(a) Test Image

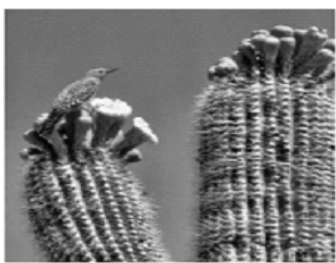

(c) CLAHE

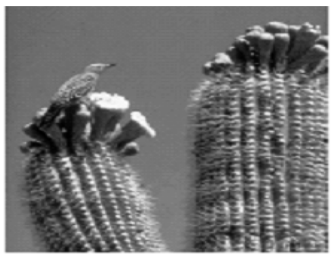

(e) Proposed

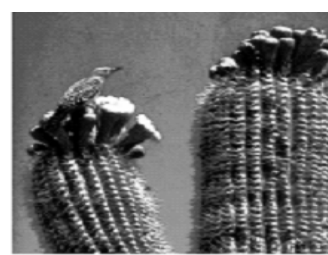

(b) $\mathrm{HE}$

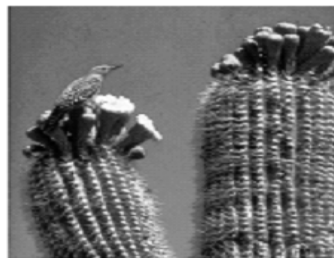

(d) $\mathrm{HMF}$

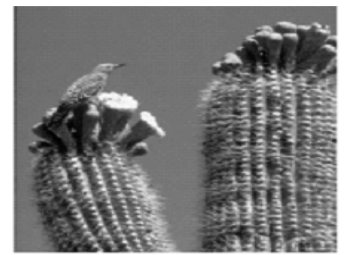

(f) Reference
Fig. 6. The results of algorithms: (b) HE, (c) ACMA, (d) HMF, (e) proposed method, (a) test image, (f) reference image

$$
P S N R=20 * \log _{10}\left(\frac{M A X i}{\sqrt{M S E}}\right)
$$

where $M A X i$ is the maximum value that the pixel image accepts. The number of pixels has a bit rate of 8 bits, $M A X i=$ 255.

The Structural Similarity Index (SSIM) is a perceptual metric that quantifies image quality degradation caused by processing, such as data compression or data loss. A comparison of the PSNRs of different enhancement algorithms (including HE, CLAHE, HMF, and the proposed method) on the 3 test images is shown in Table 1 .

Table 1

Comparison of different improvement algorithms in terms of PSNR

\begin{tabular}{|c|c|c|c|c|}
\hline & $\overline{\mathrm{HE}}$ & CLAHE & "HMF & $\begin{array}{l}\text { Proposed } \\
\text { method }\end{array}$ \\
\hline $\begin{array}{l}\text { Images } \\
\text { "Door" }\end{array}$ & 17.90 & 27.63 & 24.12 & 29.92 \\
\hline $\begin{array}{l}\text { Image } \\
\text { "Lighthouse" }\end{array}$ & 17.84 & 23.94 & 23.94 & 35.92 \\
\hline $\begin{array}{l}\text { Image } \\
\text { "Cactus" }\end{array}$ & 20.3 & 26 & 26.67 & 34.90 \\
\hline $\begin{array}{l}\text { Average } \\
\text { value }\end{array}$ & 18.68 & 25.86 & 26.84 & 33.58 \\
\hline
\end{tabular}

In Table 2, we compare SSIMs of different improvement methods. The results show that the proposed approach is superior to other improvement algorithms in terms of SSIM. The range of SSIM values extends between -1 and +1 and only equals 1 if the two images are identical. This indicates the ability to evenly improve the image with this method. 
Table 2

Comparison of different improvement algorithms in terms of SSIM

\begin{tabular}{|c|c|c|c|c|}
\hline & $\mathrm{HE}$ & CLAHE & HMF & $\begin{array}{l}\text { Proposed } \\
\text { method }\end{array}$ \\
\hline $\begin{array}{l}\text { Images } \\
\text { "Door" }\end{array}$ & 0.76 & 0.97 & 0.94 & 0.98 \\
\hline $\begin{array}{l}\text { Image } \\
\text { "Lighthouse" }\end{array}$ & 0.72 & 0.94 & 0.90 & 0.99 \\
\hline $\begin{array}{l}\text { Image } \\
\text { "Cactus" }\end{array}$ & 0.72 & 0.96 & 0.90 & 0.98 \\
\hline $\begin{array}{l}\text { Difference } \\
\text { with proposed }\end{array}$ & -0.25 & -0.02 & -0.07 & 0 \\
\hline
\end{tabular}

\section{CONCLUSIONS}

This article discusses the principles and methods of improving image contrast. A method of automatic contrast enhancement to preserve the natural appearance of the image is proposed. The main features of this method are:

- determining the weight sum of the original histogram, homogeneous histogram and Gaussian histogram as the optimal target histogram to enhance the contrast.

- use of structural measure and statistical measure of "naturalness" to determine the optimal parameters.

After comparing existing histogram-based methods with proposed variant we got these results: better by $79 \%$ than the HE method, $30 \%$ than the CLAHE method, and $25 \%$ than the HMF in the experiments for determining PSNR metric. In terms of comparing the degradation of the image(SSIM) with the reference image: described method performs better results by $25 \%$ than the HE, $2 \%$ than the CLAHE, and for $7 \%$ than HMF methods.

This means, that proposed method is better in image contrast increasing than other histogram-based methods, and it allows to find a compromise between increasing the contrast and maintaining the correct visualization.

\section{References}

[1] A. Ignatov, N. Kobyshev, R. Timofte and K. Vanhoey, "DSLRQuality Photos on Mobile Devices with Deep Convolutional Networks," 2017 IEEE International Conference on Computer Vision (ICCV), 2017, pp. 3297-3305, DOI: 10.1109/ICCV.2017.355.

[2] Gonzalez, R. C. and Woods, R. E. (2007). Digital image processing (Third Edition), ISBN: 978-0131687288.

[3] Hsu, W.-Y. and Chou, C.-Y. (2015). Medical image enhancement using modified color histogram equalization. Journal of Medical and Biological Engineering, 35(5):580-584, DOI: $10.1007 / \mathrm{s} 40846-015-0078-8$.

[4] Ponomarenko, N., Jin, L., Ieremeiev, O., Lukin, V., Egiazarian, K., Astola, J., Vozel, B., Chehdi, K., Carli, M., Battisti, F., et al. (2015). 30:57-77, ISBN: 978-82-93269-13-7.

[5] Pizer, S. M., Amburn, E. P., Austin, J. D., Cromartie, R., Geselowitz, A., Greer, T., ter Haar Romeny, B., Zimmerman, J. B., and Zuiderveld, K. (1987). Adaptive histogram equalization and its variations, 39(3):355- 368, DOI: $10.1016 /$ S0734189X(87)80186-X.

[6] Wang, Z., Bovik, A. C., Sheikh, H. R., and Simoncelli, E. P. (2004). Image quality assessment: from error visibility to structural similarity. IEEE Transactions on Image Processing, 13(4):600-612, DOI: 10.1109/tip.2003.819861.
[7] Stark, J. A. (2000). Adaptive image contrast enhancement using generalizations of histogram equalization, 9(5):889-896, DOI: $10.1109 / 83.841534$.

[8] Huang TS Fast algorithms in digital image processing / TS Huang, J.-O. Ecluid, G. J. Nussbauyer et al.; lane. with English; under ed. TS Juan Ga.: - M.: Radio and communication, 1984. 224 p, DOI: 10.4236/ami.2018.81001

[9] Frederic P. Miller, Agnes F. Vandome, John McBrewster, "Histogram equalization", VDM Publishing, 2011, 80 pages, ISBN: $6135641395,9786135641394$.

[10] Mertens, T., Kautz, J., and Van Reeth, F. (2009). Exposure fusion: A simple and practical alternative to high dynamic range photography. Computer Graphics Forum, 28(1):161-171, DOI: $10.1111 /$ j.1467-8659.2008.01171.x/

[11] Zhiming W. and Jianhua T., "A Fast Implementation of Adaptive Histogram Equalization," in Proceedings of the 8th International Conference on Signal Processing, Beijing, 2006, DOI: 10.1109/ICOSP.2006.345602.

[12] Arici, T., Dikbas, S., and Altunbasak, Y. (2009). A histogram modification framework and its application for image contrast enhancement. IEEE Transactions on Image Processing, 18(9):1921-1935, DOI: 10.1109/TIP.2009.2021548.

[13] Ross, L. and Russ, J. C. (2011). The image processing handbook. Microscopy and Microanalysis, 17(5):843, DOI: 10.1017/S1431927611012050.

[14] Yeganeh, H. and Wang, Z. (2013). Objective quality assessment of tone-mapped images. IEEE Transactions on Image Processing, 22(2):657-667, DOI: 10.1109/TIP.2012.2221725.

[15] Lisani, J.-L., Michel, J., Morel, J.-M., Petro, A. B., and Sbert, C. (2016). An inquiry on contrast enhancement methods for satellite images., 54(12):7044-7054, DOI: 10.1109/TGRS.2016.2594339

[16] Conversion between RGB and HSV, Sep, 2021 [Online]. Available: https://pws.yazd.ac.ir/latiff/teaching/DIP/12.pdf

[17] Histogram Processing, Sep, 2021 [Online]. Available: http://appliedmaths.sun.ac.za/TW793/slides/slides_3_2.pdf

[18] Peak signal to noise ratio, Aug, 2021, [Online]. Available: https://uk.wikipedia.org/wiki/PSNR

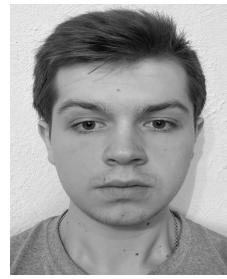

Mykola Maksymiv - a student of Lviv Polythechnic National University. In 2020 he received a bachelor's degree in computer engineering at Lviv Polytechnic National University. Now he is getting Master's Degree in the field of Computer System and Networks.

Since 2018 he has been working as a Software Engineer at Epam Systems.

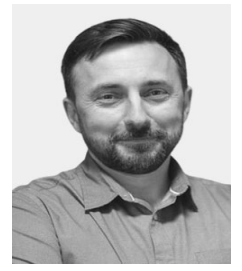

Taras Rak - Professor at Lviv Polythechnic National University, Vice-rector and Professor at IT STEP University.

A graduate of Lviv Polytechnic State University, 1996, specialty "Computer and intelligent systems and networks", honors degree. Candidate of Technical Sciences, 2005, specialty "Systems analysis and theory of optimal solutions". Since 2014, Doctor of Technical Sciences, specialty "Information Technologies". Author of over 150 scientific and educational publications. 